\title{
POSITIVE SECTIONAL CURVATURES DOES NOT IMPLY POSITIVE GAUSS-BONNET INTEGRAND ${ }^{1}$
}

\author{
ROBERT GEROCH
}

\begin{abstract}
An example is given, in dimension six, of a curvature tensor having positive sectional curvatures and negative Gauss-Bonnet integrand.
\end{abstract}

A large class of questions in differential geometry involves the relationship between the topology and the geometry of a compact Riemannian manifold. One of these is the Hopf conjecture: If, in even dimensions, the sectional curvatures of such a manifold are positive, then so is the Euler number.

The Hopf conjecture is known to be true in dimensions two and four by the following argument (Milnor, unpublished; [2]). One first writes down the Gauss-Bonnet formula, which, in every even dimension, equates the Euler number of the manifold to a certain integral over the manifold, where the integrand involves only the curvature tensor, and that only algebraically. One then shows (in dimensions two and four) that, at each point, positivity of the sectional curvatures implies positivity of this integrand. Most attempts to prove the full Hopf conjecture have been attempts to generalize this argument [1], [3], [4], [5], [6]. Thus, there arises the following, purely algebraic, question: Over a vector space of any even dimension, does a tensor having the symmetries of a curvature tensor and having positive sectional curvatures necessarily have positive Gauss-Bonnet integrand? We here answer this question in the negative.

Fix a real, six-dimensional vector space $V$. A wedge denotes the wedge product, and a star a Hodge star operator. ${ }^{2}$ Denote by $V^{2}$ the vector space of second-rank, antisymmetric tensors over $V$, by $V_{2}$ its dual (the space of 2 -forms over $V$ ), and by $V_{22}$ the vector space of symmetric linear mappings from $V^{2}$ to $V_{2}$. We shall make use of the following fact: For any element $A$ of $V_{2}$,

$$
\left((A \wedge A)^{*} \wedge(A \wedge A)^{*}\right)^{*}=\frac{2}{9}(A \wedge A \wedge A)^{*} A \text {. }
$$

For $A$ any element of $V_{2}$, denote by $T_{A}$ the element of $V_{22}$ with action

Received by the editors September 3, 1974.

AMS (MOS) subject classifications (1970). Primary 53B20.

${ }^{1}$ Supported in part by the National Science Foundation under contract GP-34721X1, and by the Sloan Foundation.

2 Our conventions for the star operation are these: For any form $A, A^{* *}=A$; for $B$ a 2 -form and $C$ a 4-form, $B\left(C^{*}\right)=C\left(B^{*}\right)=(B \wedge C)^{*}=\left(B^{*} \wedge C^{*}\right)^{*}$. Note that we introduce no metric on $V$. 
$T_{A}(X)=[A(X)] A$. Set alt $\left(T_{A}\right)=A \wedge A$, and extend the action of "alt" to arbitrary elements of $V_{22}$ by linearity.

A curvature tensor over $V$ is an element $R$ of $V_{22}$ satisfying alt $(R)=0$. For $R$ a curvature tensor, and $v$ and $v^{\prime}$ linearly independent elements of $V$, the $(v$, $\left.v^{\prime}\right)$-sectional curvature of $R$ is the number $\left[R\left(v \wedge v^{\prime}\right)\right]\left(v \wedge v^{\prime}\right)$. Set $\psi\left(T_{A}, T_{B}, T_{C}\right)$ $=\left((A \wedge B \wedge C)^{*}\right)^{2}$, and extend the action of $\psi$ to arbitrary triples of elements of $V_{22}$ by linearity. For $K$ an arbitrary element of $V_{22}$, the GaussBonnet integrand of $K$ is the number $\chi(K)=\psi(K, K, K)$. We shall give an example of a curvature tensor $R$ over $V$ with nonnegative sectional curvatures and with $\chi(R)$ negative.

The example is based on the following

LEMMA . Let $K$ be any element of $V_{22}$, and denote by $R$ the element of $V_{22}$ with action $R(X)=K(X)-\left(K^{\prime} \wedge X\right)^{*}$, where $K^{\prime}=(\operatorname{alt}(K))^{*}$. Then

$$
\chi(R)=\chi(K)-\frac{1}{2}\left[K\left(K^{\prime}\right)\right]\left(K^{\prime}\right)+\frac{2}{3}\left(K^{\prime} \wedge K^{\prime} \wedge K^{\prime}\right)^{*} .
$$

Before proving the Lemma, we construct from it the desired example.

First note that, for any $K$ in $V_{22}$, the $R$ given in the Lemma is a curvature tensor. For $K=T_{A}+T_{B}+T_{C}$, with $A, B$, and $C$ elements of $V_{2}$, this $R$ has nonnegative sectional curvatures. Choose $K$ of this form, with $A, B$, and $C$ satisfying

Condition (i). Any 6-form obtained by taking the wedge product of any three of $A, B$, and $C$, possibly with repetitions, vanishes.

We use the Lemma to evaluate $\chi(R)$. By Condition (i), $\psi$, applied to any three of $T_{A}, T_{B}$, and $T_{C}$, possibly with repetitions, vanishes; hence, the first term on the right in (2) vanishes. By (i), $T_{A}\left((A \wedge A)^{*}\right)=T_{A}\left((B \wedge B)^{*}\right)=0$, and similarly, interchanging roles of $A, B$, and $C$; hence, substituting

$$
K^{\prime}=(A \wedge A)^{*}+(B \wedge B)^{*}+(C \wedge C)^{*}
$$

into the second term, it too vanishes. By (1) and Condition (i), $(A \wedge A)^{*}$ $\wedge(A \wedge A)^{*}=0$, and similarly for $B$ and $C$; hence, substituting (3) into the third term on the right in (2), we obtain, finally,

$$
\chi(R)=4\left((A \wedge A)^{*} \wedge(B \wedge B)^{*} \wedge(C \wedge C)^{*}\right)^{*} .
$$

Thus, in order to obtain our example, we need only find elements $A, B$, and $C$ of $V_{2}$ satisfying Condition (i) together with

Condition (ii). The number $\left((A \wedge A)^{*} \wedge(B \wedge B)^{*} \wedge(C \wedge C)^{*}\right)^{*}$ is negative.

But such a system of 2 -forms is given, for example, by

$$
A=\beta \wedge \gamma+\beta^{\prime} \wedge \gamma^{\prime}, \quad B=\gamma \wedge \alpha+\gamma^{\prime} \wedge \alpha^{\prime}, \quad C=\alpha \wedge \beta+\alpha^{\prime} \wedge \beta^{\prime},
$$

where $\left(\alpha, \alpha^{\prime}, \beta, \beta^{\prime}, \gamma, \gamma^{\prime}\right)$ is any basis for the 1 -forms.

ProOF of THE LEMMA. It is convenient to introduce an index notation. A lower case Latin superscript indicates membership in $V^{2}$, a subscript membership in $V_{2}$, and a combination of superscripts and subscripts membership in the appropriate tensor product. Repeated indices, one a superscript and one a 
subscript, denote the operation of contraction. Parentheses surrounding indices denote the operation of taking the symmetric part.

Regarding " 15 " as a 0 -form, set $\epsilon^{a b c}=(15)^{*}$; regarding " 15 " as a 0-rank tensor over $V$, set $\epsilon_{a b c}=(15)^{*}$. Then each is symmetric $-\epsilon^{a b c}=\epsilon^{(a b c)}$ and $\epsilon_{a b c}=\epsilon_{(a b c)}$-and

$$
\epsilon^{a m n} \epsilon_{b m n}=\delta_{b}^{a}
$$

where $\delta_{b}^{a}$ denotes the unit tensor. For $P$ in $V_{2}, P^{*}$ is $\epsilon^{a b c} P_{c}$; for $Q$ in $V^{2}, Q^{*}$ is $\epsilon_{a b c} Q^{c}$. Equation (1) is equivalent to

$$
\epsilon^{p(a b} \epsilon^{c d) q} \epsilon_{p q m}=\frac{2}{9} \delta_{m}^{(a} \epsilon^{b c d)},
$$

for (1) results from (5) by contracting with $A_{a} A_{b} A_{c} A_{d}$.

An element $K$ of $V_{22}$ is written, in this notation, $K_{a b}$, and satisfies $K_{a b}=K_{(a b)}$. For such a $K, \chi(K)$ is given by

$$
\chi(K)=\epsilon^{a b c} \epsilon^{u v w} K_{a u} K_{b v} K_{c w},
$$

while the formulae for the $K^{\prime}$ and the $R$ in the Lemma become

$$
K^{\prime a}=\epsilon^{a p q} K_{p q}
$$

and

$$
R_{a b}=K_{a b}-\epsilon_{a b p} K^{\prime p}
$$

respectively.

From (6) and (8),

$$
\begin{aligned}
\chi(R)= & \epsilon^{a b c} \epsilon^{u v w} K_{a u} K_{b v} K_{c w}-3 \epsilon^{a b c} \epsilon^{u v w} K_{a u} K_{b v}\left(\epsilon_{c w p} K^{\prime p}\right) \\
& +3 \epsilon^{a b c} \epsilon^{u v w} K_{a u}\left(\epsilon_{b v p} K^{\prime p}\right)\left(\epsilon_{c w q} K^{\prime q}\right) \\
& -\epsilon^{a b c} \epsilon^{u v w}\left(\epsilon_{a u p} K^{\prime p}\right)\left(\epsilon_{b v q} K^{\prime q}\right)\left(\epsilon_{c w r} K^{\prime r}\right) .
\end{aligned}
$$

We wish to evaluate each of the four terms on the right in (9). Leave the first term unchanged. For the second term, contract (5) with $K_{a b} K_{c d} K^{\prime m}$ and use (7) to obtain

$$
\epsilon^{a c p} \epsilon^{b d q} K_{a b} K_{c d} \epsilon_{p q m} K^{\prime m}=\frac{1}{3} K_{a b} K^{\prime a} K^{\prime b}-\frac{1}{2} \epsilon_{a b c} K^{\prime a} K^{\prime b} K^{\prime c} .
$$

For the third term, first contract (5) with $\epsilon_{c d n}$ and use (4):

$$
\epsilon^{p c a} \epsilon^{q d b} \epsilon_{p q m} \epsilon_{c d n}=\frac{1}{6} \delta_{m}^{(a} \delta_{n}^{b)}-\frac{1}{3} \epsilon^{a b p} \epsilon_{m n p} .
$$

Next contract (11) with $K_{a b} K^{\prime m} K^{\prime n}$ and use (7) to obtain

$$
\epsilon^{\epsilon q d b} \epsilon_{p q m} \epsilon_{c d n} K_{a b} K^{\prime m} K^{\prime n}=\frac{1}{6} K_{a b} K^{\prime a} K^{\prime b}-\frac{1}{3} \epsilon_{a b c} K^{\prime a} K^{\prime b} K^{\prime c} .
$$

For the fourth term, first contract (11) with $\epsilon_{a b s}$ and use (4): 


$$
\epsilon^{p c a} \epsilon^{q d b} \epsilon_{p q m} \epsilon_{c d n} \epsilon_{a b s}=-\frac{1}{6} \epsilon_{m n s} .
$$

Then contract (13) with $K^{\prime m} K^{\prime n} K^{s}$ :

$$
\epsilon^{p c a} \epsilon^{q d b} \epsilon_{p q m} \epsilon_{c d n} \epsilon_{a b s} K^{\prime m} K^{\prime n} K^{\prime s}=-\frac{1}{6} \epsilon_{a b c} K^{\prime a} K^{\prime b} K^{\prime c} .
$$

Finally, substituting (10), (12), and (14) into (9), we obtain

$$
\chi(R)=\epsilon^{a b c} \epsilon^{u v w} K_{a u} K_{b v} K_{c w}-\frac{1}{2} K_{a b} K^{\prime a} K^{\prime b}+\frac{2}{3} \epsilon_{a b c} K^{\prime a} K^{\prime b} K^{\prime c},
$$

which is precisely (2). This completes the proof of the Lemma.

We remark that one obtains immediately from this example both (i) examples in all even dimensions six or greater, and (ii) examples of curvature tensors with positive sectional curvatures and negative Gauss-Bonnet integrand. Equation (1) can be generalized to higher even dimensions. Can (2)? Of course, the problem of actually realizing such an example on a compact Riemannian manifold is more difficult.

I wish to thank John Thorpe and, especially, Ann Stehney for helpful discussions on this problem.

\section{REFERENCES}

1. R. L. Bishop and S. I. Goldberg, Some implications of the generalized Gauss-Bonnet theorem, Trans. Amer. Math. Soc. 112 (1964), 508-535. MR 29 \# 574.

2. S. S. Chern, On curvature and characteristic classes of a Riemann manifold, Abh. Math. Sem. Univ. Hamburg 20 (1955), 117-126. MR 17, 783.

3. A. Gray, The six-dimensional Gauss-Bonnet integrand (to appear).

4. H. Samelson, On curvature and characteristic classes of homogeneous spaces, Michigan Math. J. 5 (1958), 13-18. MR 21 \#2277.

5. J. A. Thorpe, Some remarks on the Gauss-Bonnet integral, J. Math. Mech. 18 (1969), 779-786. MR 41 \#963.

6. A. Weinstein, Remarks on curvature and the Euler integrand, J. Differential Geometry 6 (1971/72), 259-262. MR 45 \#7641.

Department of Mathematics, University of Chicago, Chicago, Illinois 60637 